\title{
Detection of Concentric Circles for Camera Calibration
}

\author{
Guang JIANG and Long QUAN \\ Department of Computer Science \\ Hong Kong University of Science and Technology \\ Kowloon, Hong Kong \\ \{gjiang,quan\}@cs.ust.hk
}

\begin{abstract}
The geometry of plane-based calibration methods is well understood, but some user interaction is often needed in practice for feature detection. This paper presents a fully automatic calibration system that uses patterns of pairs of concentric circles. The key observation is to introduce a geometric method that constructs a sequence of points strictly convergent to the image of the circle center from an arbitrary point. The method automatically detects the points of the pattern features by the construction method, and identify them by invariants. It then takes advantage of homological constraints to consistently and optimally estimate the features in the image. The experiments demonstrate the robustness and the accuracy of the new method.

Keywords: camera calibration, concentric circles, cross ratio, homological constraint.
\end{abstract}

\section{Introduction}

Using planar patterns [21, 18, 19] for camera calibration is popular for its practical convenience. Such calibration methods consist of three major steps: detection and location of pattern features in image, identification of features with the known calibration pattern in space, and the computation of camera parameters using the feature correspondences. Different planar patterns are introduced, e.g. grid pattern $[21,18,19]$, circular pattern $[9,3,11,6,20]$ and some mixed pattern [13]. However, most of the works have been concentrated on the geometric aspect, i.e. deriving new constraint for the computation of camera parameters. The bottleneck of automatic detection and identification of the features for the practice of calibration has not been put sufficient efforts.

Features on a planar pattern are usually detected by corners detection algorithm (Harris operator etc.) or edge detection algorithm (Canny operator etc.) or lines or conics detection algorithm (Hough Transform etc.) or their combinations. All these methods are often time-consuming, more importantly, the identification of the image features with the known pattern features in space is difficult, and often some degrees of user interaction is required. This is particularly true for point features even many ad hoc correspondence methods have been developed for specifically designed patterns [13]. A good pattern for calibration should first provide sufficient geometric constraints, then should be easily detectable and identifiable.

We will use planar pattern of pairs of concentric circles to develop our approach in this paper as this pattern is geometrically richer than point-like features. Some geometric properties of such pattern have been introduced and discussed by researchers $[11,6,20]$, but their geometric derivation could have been much simplified by using the simple fact that the image of a pair of concentric circle intersects at a repeated pair of points, the image of the circular points [8]. Moreover, these papers have used the Hough Transform based method to detect the conics that are the images of the circles without considering the intrinsic special geometric properties of the pattern. These papers also do not consider the important feature identification issue.

This paper is not about new geometry for calibration, instead, we will introduce in Section 3 an interesting constructive geometric method that one point approaches the center of circle in a convergence manner. We will use this construction tool to lay down a unified method with detection, location and identification of these patterns in an image in Section 4 and Section 5. Section 6 presents the homological constraints to obtain optimal and consistent estimation of image features.

The main contributions may be summarized as follows:

- It introduces a constructive method for the image of the center of the circles from a pair of conics.

- It automatically detect and identify the image points with the calibration patterns in a robust and efficient manner.

- It enforces the homological constraints for consistent and optimal location of the pattern features.

- It presents a complete system of practical and automatic calibration method. 
The method will be summarized in Section 7 and experimented in Section 8. A short conclusion is given in Section 9.

\section{Basic notations}

Throughout the paper, a scalar or a geometric object is denoted by a normal letter, while a row vector is by a small case letter in bold, and a matrix by a capital letter in bold.

Camera Model A camera is always modelled by a perspective projection $[5,8]$. The projection may be represented by a $3 \times 4$ projection matrix $\mathbf{P}$ such that $\mathbf{P}=\mathbf{K}[\mathbf{R} \mid \mathbf{t}]$, where $\mathbf{R}$, a $3 \times 3$ rotation matrix and $\mathbf{t}$, a $3 \times 1$ translation vector, are extrinsic parameters and

$$
\mathbf{K}=\left[\begin{array}{ccc}
a f & s & u_{0} \\
0 & f & v_{0} \\
0 & 0 & 1
\end{array}\right]
$$

is intrinsic parameters matrix. The image of the absolute conic $\omega$ is by definition $\lambda \omega=\left(\mathbf{K K}^{T}\right)^{-1}$.

Harmonic conjugate points Given collinear points $A$, $A^{\prime}, P$ and $Q$, the points $A$ and $A^{\prime}$ are harmonic conjugate points with respect to $P$ and $Q$ if the cross ratio $\left\{A, A^{\prime} ; P, Q\right\}=-1$, (cf. [17]). There is an important special case when $P$ and $Q$ divide segment $A A^{\prime}$ internally and externally in the ratio $1: 1$. In this case $P$ is the midpoint and $Q$ is the point at infinity on the line.

Homology A homology $[17,8]$ is a special projective transformation that arises in some special imaging situations [4]. It can be parameterized [16, 8] by a line of fixed points $\mathbf{l}$ (axis), a fixed point not on the axis $\mathbf{v}$ (vertex), and a scalar factor $\mu$, its characteristic ratio.

$$
\mathbf{G}=\mathbf{I}_{3 \times 3}+\mu \frac{\mathbf{v} \mathbf{l}^{T}}{\mathbf{v}^{T} \mathbf{l}},
$$

where $\mathbf{I}_{3 \times 3}$ is $3 \times 3$ identity matrix.

\section{The basic idea of the new method}

\subsection{A simple geometric observation}

Given a circle as illustrated in Figure 1, we will try to reach the center $O$ of circle from an arbitrary point $P$ inside the circle. First draw an arbitrary line through the point $P$, take the midpoint $P_{1}$ of the chord generated by the line. Then, do the same by drawing another arbitrary line through the point $P_{1}$, and take the midpoint $P_{2}$ of the chord generated by the new line. It is immediate to observe that

$$
\left|O P_{2}\right|<\left|O P_{1}\right| \text {. }
$$

The point $P_{2}$ is always closer to the center $O$ than the previous point $P_{1}$. Continuing this construction results in a sequence points that strictly converge at the center of the circle as long as each time a different line is drawn. In other words, the center of the circle is always in a strictly decreasing convex area formed by all the chords.

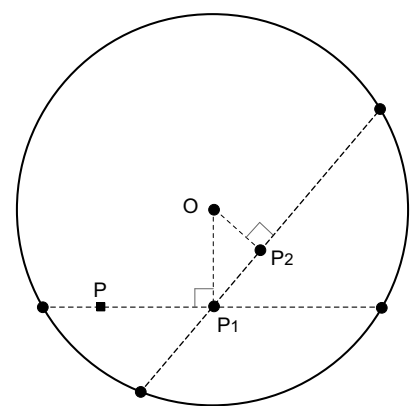

Figure 1: Construction of a sequence of points $P_{1}, P_{2}, \ldots$ strictly convergent to the center of the circle from any point $P$.

This construction is for a circle in a Euclidean space, we need to move this construction into conics in a projective plane.

\subsection{Construction of the image of the mid- point}

For our pattern of a pair of concentric circles, its image is a pair of conics. The goal is to construct the image of the center of circles from the observation of the pair of conics. The key is to translate the Euclidean concept of the midpoint of a given segment into a projective notion of harmonic conjugate that could be determined by a pair of conics.

In space, for a pair of concentric circles, a line cuts it in the two points $A, A^{\prime}$ of the outer circle, and the two points $B, B^{\prime}$ of the inner circle. There are two midpoints of segments of $\overline{A A^{\prime}}$ and $\overline{B B^{\prime}}$ : the finite midpoint $P$ and the infinite point $Q$ on the line at infinity as illustrated in Figure 2. In projective terms of cross-ratios, $P$ and $Q$ are the internal and external harmonic division of the segment $\overline{A A^{\prime}}$ and $\overline{B B^{\prime}}$, and they are called the harmonic conjugate points with respect to each pair of points on each circle:

$$
\left\{A, A^{\prime} ; P, Q\right\}=\left\{B, B^{\prime} ; P, Q\right\}=-1 .
$$

Now project this pair of concentric circles onto an image plane as shown in Figure 3. Since the harmonic division is projective invariant that is preserved in the image, i.e. we still have two equations

$$
\left\{\mathbf{a}, \mathbf{a}^{\prime} ; \mathbf{p}, \mathbf{q}\right\}=\left\{\mathbf{b}, \mathbf{b}^{\prime} ; \mathbf{p}, \mathbf{q}\right\}=-1,
$$

where $\mathbf{a}, \mathbf{a}^{\prime}, \mathbf{b}$ and $\mathbf{b}^{\prime}$ are the image of the intersection points $A, A^{\prime}, B$, and $B^{\prime}$ that are measurable in the image, the two 


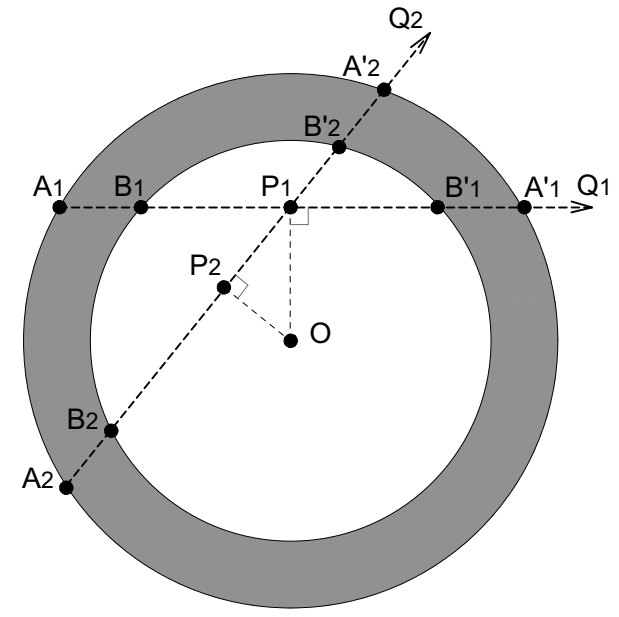

Figure 2: The geometry of the midpoints of concentric circles in space.

equations result a quadratic equation whose two solutions are the pair of points $\mathbf{p}$ and $\mathbf{q}$. In image space, we know the inside convex sets of the segment $\overline{\mathbf{a a}}{ }^{\prime}$, we therefore choose the midpoint $\mathbf{p}$ form the solution pair to be that is inside the segment $\overline{\mathbf{a a}}$.

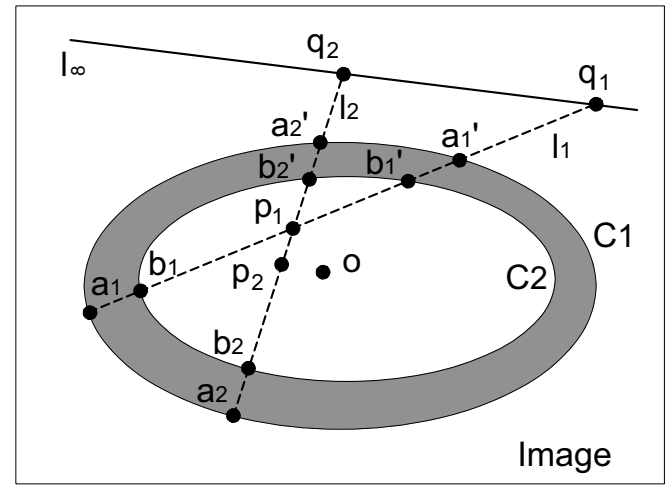

Figure 3: The construction of the image of the midpoint in an image plane

\subsection{Construction of the image of center of cir- cles}

With this construction method of the image of the midpoint from a pair of conics, we could, from an arbitrary point $\mathbf{p}$ on the plane, construct a sequence of points rapidly approaching the image of the center of circles: From an arbitrary point, draw a line, get the intersection points with the conics and construct the image of the midpoint $\mathbf{p}_{1}$ by the harmonic ratio, then draw another line from $\mathbf{p}_{1}$ and continue.

The sequence of points $\mathbf{p}_{i}$ strictly converges to the image of the center of the circles as back in space, we have

$$
\left|O P_{1}\right|>\left|O P_{2}\right|>|\cdots>| O P_{i-1}|>| O P_{i} \mid
$$

\section{Detection by construction}

Armed with this construction tool of the image of the midpoint and the image of the center of circle, we describe how this construction is combined with an image point detector to achieve an automatic detection approach.

Making starting points In principal, we can start from a random point in the image plane and draw a random line through it. We could achieve our construction if the random line intersects the pair of conics. Otherwise, another point or another line is randomly generated.

Our current strategy is to use some 'seed' points to start the construction procedure. The seed points are drawn from a regular square grid on the image. The grid size is determined by the size of the pattern so that at least one grid point is falling inside each pattern for ease of construction. One example is given in Figure 4. For the random generation of lines, we just alternatively take the horizontal and vertical lines, which is both convenient and efficient.

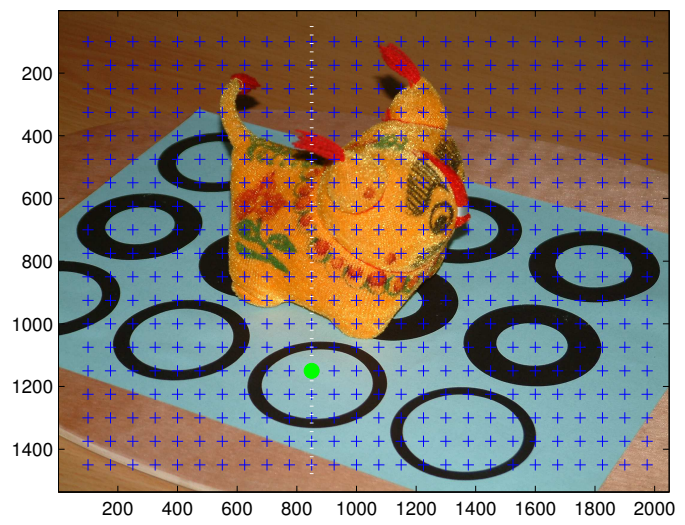

Figure 4: A calibration pattern in a scene and the grid points marked as '+' that are used as seed points to start the construction procedure. A vertical line in white is drawn from a seed point.

Detecting feature points in $1 D$ images The advantage of the approach lies in that we do not need to pre-detect corner points or whatever edge points at the beginning. Our operations are restricted to a line, so we can take all pixels along that line to form our 1D image as illustrated in Figure 5. Then, it is only necessary to detect 4 point features beside 
'seed' point from this 1D image. This is far more efficient, accurate and robust compared to using a usual 2D edge detector. The image line is convoluted with a derivative of 1D Gaussian kernel [12]. The edge points are the local maxima of the convoluted signal above a threshold.

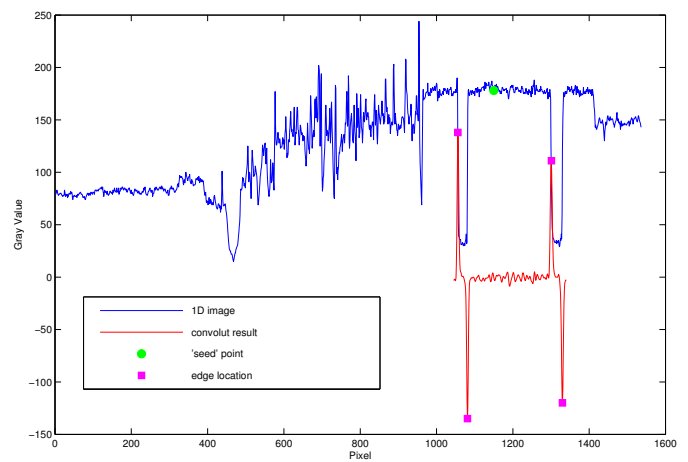

Figure 5: The 4 feature points are the edge points beside the 'seed' point of this 1D signal and could be easily detected. Note that we needn't to search the whole line.

Checking the set of points by invariant For the current point $\mathbf{p}_{i-1}$ and the current line, the nearest 2 points on the line on each side of the current point are selected to form a set of 4 ordered points. The harmonic conjugate points $\mathbf{p}_{i}$ and $\mathbf{q}_{i}$ are constructed from the 4 points. Then we define the cross ratio

$$
\rho_{i}=\left\{\mathbf{b}_{i}^{\prime}, \mathbf{a}_{i}^{\prime} ; \mathbf{p}_{i}, \mathbf{q}_{i}\right\}
$$

If the set of 4 ordered points are indeed the intersection points with the pair of conics, its associated cross-ratio $\rho_{i}$ forms a strictly increasing sequence:

$$
\rho_{1}<\cdots<\rho_{i-1}<\rho_{i}<\cdots=\frac{r_{1}}{r_{2}}
$$

and converges to the ratio of the radii of the circles. This is due to the fact that the corresponding chord on the circle is strictly increasing to reach the diameter of the circle as the midpoint of the chord is approaching to the center of the circle.

By tracking this cross-ratio during the construction, the starting points that are not falling inside the inner conic area will be eliminated.

At the end, this procedure not only constructs the image of the center $\mathbf{o}$ of the circles, but also detects the points $\mathbf{q}_{i}$ of the vanishing line $\mathbf{l}_{\infty}$, and the points $\mathbf{a}_{i}, \mathbf{a}_{i}^{\prime}$ and $\mathbf{b}_{i}$ and $\mathbf{b}_{i}^{\prime}$ on the two conics.

\section{Identification by invariant}

Most point-based calibration methods can not automatically identify the image corner points to the points of the calibration pattern in space. Interactive manipulation is always necessary, for instance, it is necessary to interactively mark up 4 points for plane-based calibration methods [21, 18].

The configuration of a pair of concentric circles is projected onto a pair of conics. It is well-known [14] that there are two projective invariants $\operatorname{Trace}\left(\mathbf{C}_{1}^{-1} \mathbf{C}_{2}\right)$ and $\operatorname{Trace}\left(\mathbf{C}_{2}^{-1} \mathbf{C}_{1}\right)$ associated with a pair of conics. It is straightforward to see that these two invariants are equivalent for a pair of concentric circles as it results in a degenerate configuration that they are intersecting onto a repeated pair of points instead of 4 independent points.

We can use this invariant in its algebraic form, but more simply, this invariant is equivalent to the following crossratio

$$
\rho=\left\{\mathbf{b}^{\prime}, \mathbf{a}^{\prime} ; \mathbf{o}, \mathbf{p}\right\}=\{\mathbf{b}, \mathbf{a} ; \mathbf{o}, \mathbf{p}\}=\frac{r_{1}}{r_{2}},
$$

where $\mathbf{o}$ is the imaged circle center, $\mathbf{a}$ and $\mathbf{b}$ are the intersection points of a line through $\mathbf{o}$ with the circles, and $\mathbf{p}$ is the vanishing point of that line. We prefer using this cross-ratio as it is straightforward and the quantities $\mathbf{o}$ and $\mathbf{p}$ are already available by our construction method.

This independent projective invariant is sufficient to uniquely identify a pair of concentric circles provided that the radii ratio differ.

\section{Optimal Location by homological constraints}

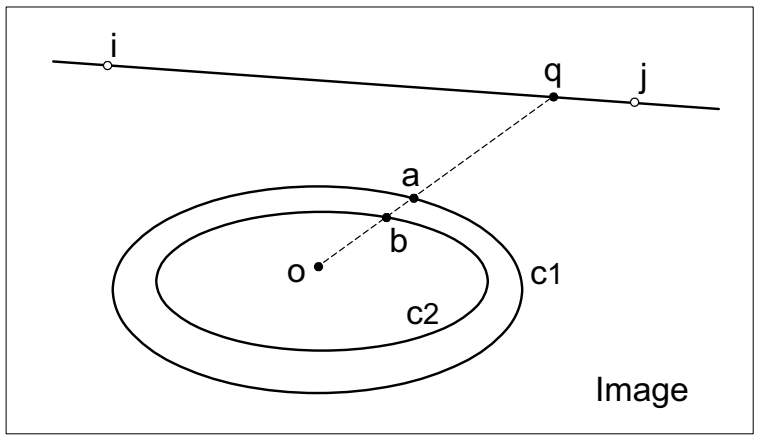

Figure 6: Optimal and consistent estimation of a pair of conics being image of a pair of concentric circles.

Usually, each conic of the pair is to be determined independently, however this may result in a pair of conics that is not consistent with the image of a pair of concentric circles. For instance, a pair of conics from a pair of concentric circles should still intersect only in a repeated pair of 
points like a pair of concentric circles (they intersect in the repeated pair of circular points) as this incidence property is preserved by the projection.

Here in addition to all points of each conic at our disposition, all points on the vanishing line, and the image of the center of circles are already available. We will take advantage of intrinsic constraints to optimize the location of all these geometric quantities consistently and more accurately, which is also the key to the accuracy of the final calibration results.

It is known and easy to see that there exists a homological relationship between points of the two conics [17, 8]. Previous researchers did not exploit this geometric constraint for estimation purpose.

A line through the image of the center of circles $\mathbf{o}$ and a point $\mathbf{q}$ on the vanishing line $\mathbf{l}_{\infty}$ intersects the pair of conics in two points $\mathbf{a}$ and $\mathbf{b}$ on the same side of the image of the center of circles. The points $\mathbf{a}$ and $\mathbf{b}$ satisfy a homology $\mathbf{G}, \lambda \mathbf{b}=\mathbf{G a}$, with its fixed line being the vanishing line $\mathbf{l}_{\infty}$ and its fixed point the image of the center of circles $\mathbf{0}$. Therefore, the two conics are related by the homology as:

$$
\lambda \mathbf{C}_{2}=\mathbf{G}^{-T} \mathbf{C}_{1} \mathbf{G}^{-1} .
$$

The homology can be parameterized $[16,8]$ by its fixed line, fixed point and a cross-ratio:

$$
\mathbf{G}=\mathbf{I}_{3 \times 3}+\rho \frac{\mathbf{o} \mathbf{l}_{\infty}^{T}}{\mathbf{o}^{T} \mathbf{l}_{\infty}}
$$

where $\mathbf{I}_{3 \times 3}$ is $3 \times 3$ identity matrix.

The pole-polar relationship [17] between the image of the center of circles $\mathbf{o}$ and the vanishing line $\mathbf{l}_{\infty}$ with respect to the outer conic $\mathbf{C}_{1}$

$$
\lambda \mathbf{l}_{\infty}=\mathbf{C}_{1} \mathbf{o},
$$

still holds.

Equations 5, 6 and 7 are the intrinsic constraints on the redundant conics, image of center and the vanishing line. For a pair of constrained conics, it can be minimally parameterized by 7 parameters, for instance, we can choose the 5 parameters of the outer conic and the 2 parameters of the vanishing line as the pole-polar relationship could be substituted into the homology to minimize

$$
f\left(\mathbf{C}_{1}, \mathbf{l}_{\infty}\right)=\sum d^{2}\left(\mathbf{a}, \mathbf{C}_{1}\right)+d^{2}\left(\mathbf{b}, \mathbf{C}_{2}\right)
$$

where $\mathbf{C}_{2}$ is parameterized by $\mathbf{C}_{1}$ and $\mathbf{l}_{\infty}$. It can be easily solved using Levenberg-Marquart algorithm either for a real distance or an approximate distance function [1] as the initial estimates are already very good.

For all pairs of constrained conics available in an image, the vanishing line is common to all pairs, so each pair is just parameterized by one of the conics, we can minimize

$$
\sum f_{i}\left(\mathbf{C}_{i}, \mathbf{l}_{\infty}\right)
$$

over the number of the pairs of conics.

The planar homography is estimated from the correspondences of the pair of circular points and the centers of circles. In image plane, each image of the center of the conics is determined as the pole of the vanishing line with respect to each conic.

Once we obtained an optimized solutions to the image of the circular points and the homography, the intrinsic and extrinsic parameters are extracted in the same way as the plane-based method $[21,18]$ and all camera parameters are globally optimized using Maximum Likelihood Estimation method.

\section{Method outline}

Now we can describe the complete calibration procedure.

1. Preparation of the patterns Prepare a planar pattern of pairs of concentric circles. The position of the circle center and the radius of each circle is known. At least two pairs are necessary, take images of this planar pattern, at least 3 images are necessary. This is the only manual part of the calibration. After this, the following steps are completely automated.

\section{Detection and identification of pattern features}

(a) Generate the seed points in the image plane by drawing a regular square grid. The size of the grid is fixed and depend on the smallest area of the image of the inner circle may be occurred in practice. So that there is at least one grid point falling inside the any imaged pair of concentric circles.

(b) Start the construction method from each seed point, and each seed point is to be either discarded if it is outside the inner conics or converged to the position of the image of the circle center.

(c) Each pair of conics is identified by its unique invariant cross-ratio.

\section{Location optimization}

(a) Optimize the geometry of each pair of conics using a homology-based minimal parametrization.

(b) Optimize all pairs of conics and the homography between the image plane and the calibration pattern.

4. Extraction of intrinsic and extrinsic parameters The parameters are extracted from the circular points and the homography as proposed in previous planebased calibration method $[21,18]$. 


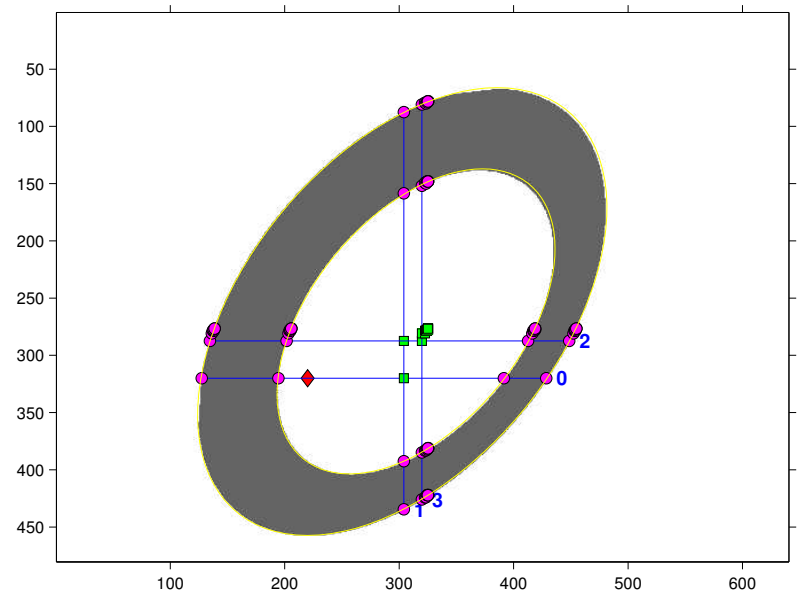

Figure 7: Different steps of the construction of the image of the center of the circles from a pair of conics with simulated data. The constructed images of the midpoints are marked with a square. The point in diamond shape is the starting point.

\section{Experimental results}

The method has been implemented and tested on both simulated and real image data. The whole system will be put into public domain. As we have stressed that here it does not make sense to compare with other calibration methods as they are all geometrically equivalent. We are solving two problems of practical importance for calibration: the automatic detection of features vs. user interaction and the accurate estimate of the features in image.

We have first set up a simulated experiment to validate our approach as illustrated in Figure 7. About 5 steps, the procedure converges to the image of the center of circles up to pixel accuracy.

It should be noted from Figure 7 that the image of the center of circles DOES NOT the center of the conics and cannot be detected using the general moment based methods[7, 10]. Heikkilä and Silven[10] used a recursive procedure with the camera parameters to correct the bias between the imaged center of circles and center of conics after knowing the latter positions. However, in our method, we can directly locate the positions of imaged center of circles without considering the camera parameters.

We then did real experiments to show the robustness and accuracy of the detection. The seed points in images are marked with ' + '. If a seed point does converge to the position of the imaged circle center, it is drawn as a diamond marker and the position of the center as a ' $x$ ' marker. When more than one pair of concentric circles is present, it is identified by a numerical label at the center. Figure 8 shows three images of an experiment of a pattern of one pair of concentric circles under different poses and backgrounds. It shows that every seed point inside the conics does converge to the center while all other seed points are soon discarded due to its divergency.

Figure 9 shows one experiment of a pattern of 6 pairs of concentric circles. The imaged circle centers are successfully located and identified. The ratios of inner and outer radii varies from 0.50 to 0.75 with a interval of 0.05 . The experiment results show that the absolute errors of the ratios are less than 0.005 .

Figure 10 shows one experiment of a pattern of 12 pairs of concentric circles with a toy on it. This shows that our method is very efficient at detecting a complete pair of concentric circles, however it does not detect any pair that is even partially occluded. This does not limits our method for the calibration task and it can be improved later. This experiment includes 19 images, and these images are captured from different positions related to the pattern with fixed intrinsic parameters. Combining our feature detection with Zhang's [21] calibration method, we get the camera matrices related to these 19 images. These matrices are used to create the visual hull $[2,15]$ of the toy in order to verify the calibration results. Views of the 3D model with the cameras' positions and the pattern are shown in Figure 11. The interested reader can also integrate our feature detection with Heikkilä's [9] or other well studied calibration methods.

\section{Conclusion}

In this paper, we present an automatic calibration method that makes the calibration task practical as all detection, location and identification of pattern features are fully automatic. The proposed pattern is a planar one with variable number of pairs of concentric circles. The key is the introduction of a simple constructive geometric method to approximate the image of the circle center from an arbitrary point. We hope that method can be easily used by any layman to easily calibrate his camera.

\section{Acknowledgments}

This project is partially supported by the Hong Kong RGC Grant HKUST 6182/04E.

\section{References}

[1] F. Bookstein, Fitting conic sections to scattered data, Computer Vision, Graphics and Image Processing, vol. 9, pp. 5671, 1979.

[2] C.H. Chien and J.K. Aggarwal: Identification of 3D objects from multiple silhouettes using quadtrees/octrees. Computer Vision, Graphics and Image Processing 36, 1986, pp.256273. 

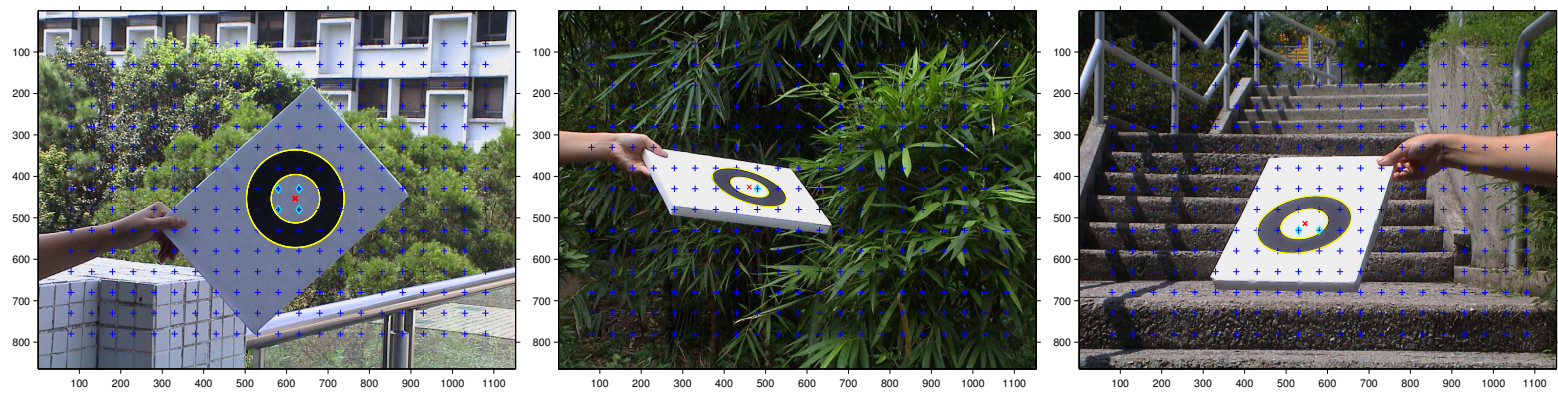

Figure 8: The experiment with a pattern of one pair of concentric circles. The seed points are shown in '+', convergent seed points in diamond, and the final imaged circle centers in ' $x$ '.
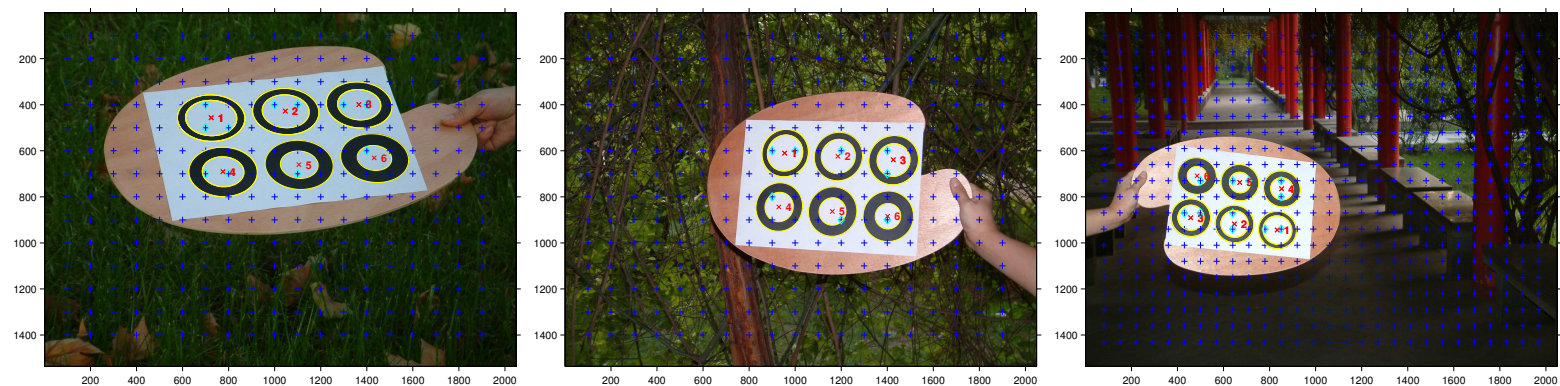

Figure 9: The experiment with a pattern of 6 pair of concentric circles. Each pair is identified with a numerical label. The seed points are shown in ' + ', convergent seed points in diamond, and the final imaged circle centers in ' $\times$ '.

[3] Q. Chen, H. Wu and T. Wada, Camera Calibration with Two Arbitrary Coplanar Circles, European Conf. Computer Vision, 2004

[4] A. Criminisi, Accurate Visual Metrology from Single and Multiple Images, $\mathrm{PhD}$ Thesis, University of Oxford

[5] O.Faugeras, Three-Dimensional Computer Vision: A Geometric Viewpoint, MIT Press , 1993.

[6] V. Fremont, R. Chelalli, Direct Camera Calibration using Two Concentric Circles from a Single View, International Conference on Artificial Reality and Telexistence, Tokyo, 2002

[7] L. O'Gorman, A.M. Bruckstein, C.B. Bose, I. Amir, Subpixel registration using a concentric ring fiducial, 10th International Conference on Pattern Recognition, vol.2, pp. 16-21, 1990.

[8] R. Hartley and A. Zisserman: Multiple View Geometry in Computer Vision, Cambridge University Press, 2000.

[9] J. Heikkilä, Geometric camera calibration using circular control points. IEEE Transactions on Pattern Analysis and Machine Intelligence, 22(10), pp.1066-1077, 2000.

[10] J. Heikkilä and O. Silven, A Four-Step Camera Calibration Procedure with Implicit Image Correction. IEEE Computer Vision and Pattern Recognition, pp. 1106-1112, 1997.

[11] J.S. Kim , H.W. Kim and I.S. Kweon, A Camera Calibration Method using Concentric Circles for Vision Applications, ACCV pp. 23-25, 2002.

[12] D. Marr and E. Hildreth, Theory of edge detection, Proc. of Royal Society of London B-207, pp. 187-217, 1980.
[13] C. Matsunaga, Y. Kanazawa and K. Kanatani, Optimal grid pattern for autmated camera calibration using cross ratio, IEICE Transactions on Fundamentals, Vol. E83-A, No. 10, pp. 1921-1928, 2000

[14] J.L. Mundy and A.P. Zisserman, editors. Geometric Invariance in Computer Vision.MIT Press, Cambridge, MA, 1992.

[15] W.Niem: Robust and Fast Modelling of 3D Natural Objects from Multiple Views, Proc. SPIE, Vol. 2182, pp. 388-397, 1994.

[16] M. Pollefeys and L. Van Gool, A Stratified Approach to Metric Self-Calibration, Computer Vision and Pattern Recognition, pp. 407-412, 1997.

[17] J. Semple, and G. Kneebone: Algebraic Projective Geometry. Oxford University Press, 1952.

[18] P. Sturm and S. Maybank, On Plane-Based Camera Calibration: A General Algorithm, Singularities, Applications. IEEE Conf. on Computer Vision and Pattern Recognition, pp. 432437, 1999.

[19] B.Triggs, Autocalibration from Planar Scenes, Proc. Fifth European Conf. Computer Vision, pp. 89-105, 1998.

[20] C. Yang, F. Sun, Z. Hu, Planar Conic Based Camera Calibration, ICPR, 2000.

[21] Z. Zhang, A flexible new technique for camera calibration, IEEE Trans. on Pattern Analysis and Machine Intelligence, 22(11), pp.1330-1334,2000. 

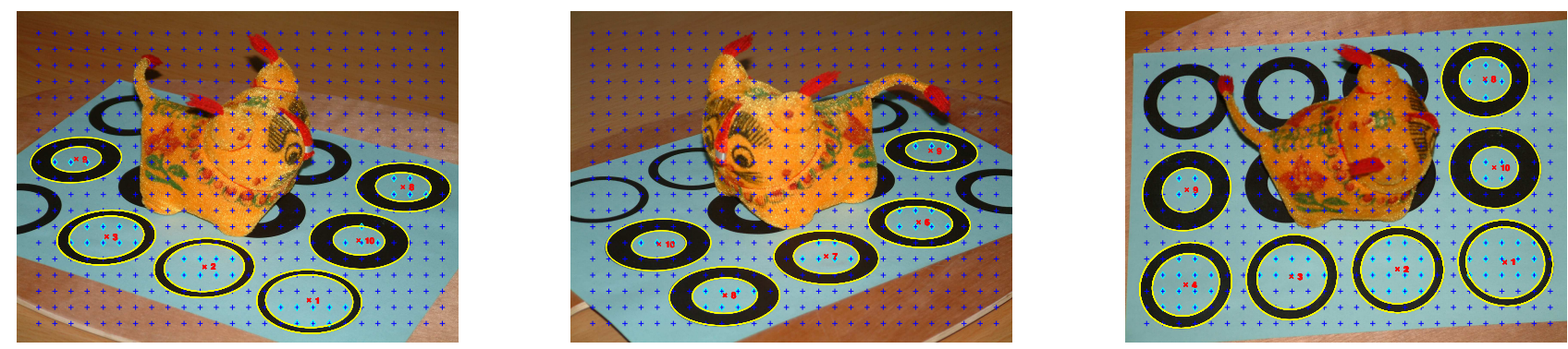

Figure 10: 3 images of one image sequence with fixed internal parameters used for a 3D reconstruction.
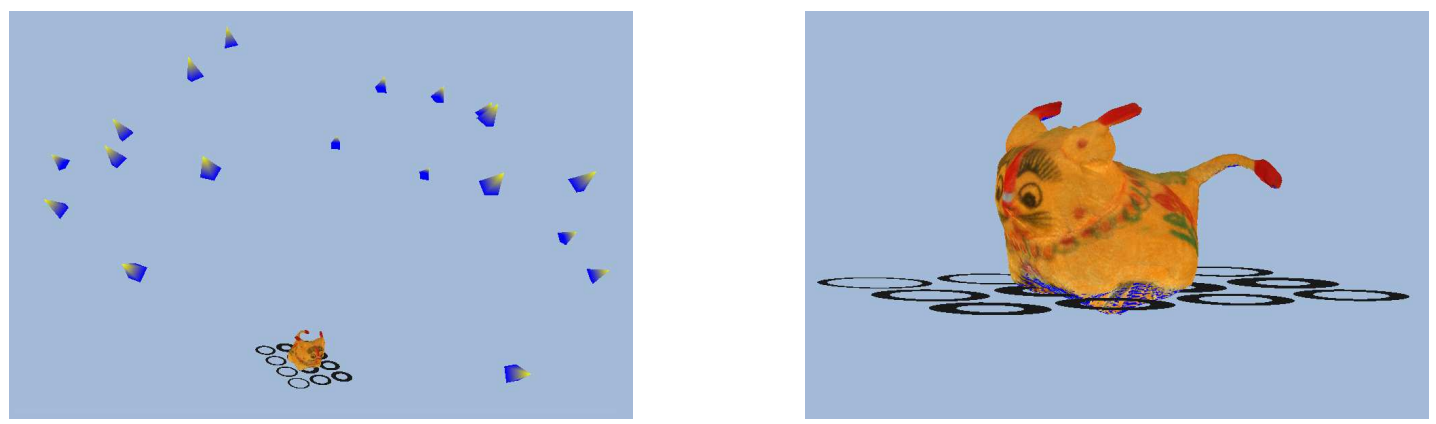

Figure 11: Reconstruction results of the toy with the camera poses and pattern. 